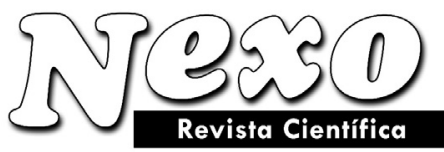

ISSN 1818-6742

Impreso en Nicaragua.

www.nexo.uni.edu.ni

Vol. 23, No. 02, pp.41-46/Noviembre 2010

\title{
Programación del riego de la papa en el "Valle del Yabú", Santa Clara, Cuba.
}

\author{
J. Pacheco ${ }^{*}$, A. Pérez y B. Job \\ Universidad Central "Marta Abreu" de Las Villas, C. Camajuani Km. 5 Santa Clara, Cuba. \\ e-mail: juanps@uclv.edu.cu, apcorra@uclv.edu.cu, bolema@uclv.edu.cu
}

(recibido/received: 08-Julio-2010; aceptado/accepted: 08-Octubre-2010)

\section{RESUMEN}

El objetivo del presente trabajo, ejecutado durante dos cosechas 2009 y 2009-10 fue estudiar la programación del riego de la papa que se realiza en la Unidad Cooperativa No.2 del "Valle del Yabú" de Santa Clara y realizar correcciones para lograr el establecimiento de una programación de riego científicamente fundamentada. En la primera cosecha, se comprobó que el momento de aplicación del riego y el volumen de agua aportado no obedecían a criterios técnicos, sino que dependían de decisiones empíricas de las personas que se relacionaban con esta actividad y la programación del riego se caracterizaba por aplicar muy pequeñas láminas de agua que no lograban humedecer $20 \mathrm{~cm}$ de profundidad de suelo, de modo que, el riego obstaculizaba las labores fitosanitarias y culturales y dejaba el suelo con déficit hídrico. En ambas cosechas, se colocaron tensiómetros a 20 y $40 \mathrm{~cm}$ de profundidad del suelo, se tomaron muestras de humedad del mismo periódicamente y se efectuó un balance diario del agua en el suelo, con lo cual, se disminuyó el número de irrigaciones durante la segunda cosecha.

Palabras claves: Balance hídrico, régimen de riego, pivote central.

\begin{abstract}
The objective of the current research was to study the applied irrigation scheduling of the potato during two crop cycles (2009 -2009/10) in Cooperative No. 2 in the Yabú Valley of Santa Clara. Also to carry out corrections required in order to establish a scientifically based irrigation program. In the first crop cycle it was proven that the timing of irrigation application and the amount of water applied did not meet the technical criteria but was dependant on the decisions taken by persons with practical experience in relation to this activity and the programming of irrigation characterized by the application of small depths of irrigation which did not moisten below $20 \mathrm{~cm}$ of soil depth in a way which the irrigation interfered with the cultural and chemical processes and left the soil with a hydric deficit. In both crops tension meter were placed at 20 and 40 soil depth, taking periodic measurement of humidity and daily measurements of soil and balance water, because of this the number of irrigation was reduce during the second crop cycle.
\end{abstract}

Key words: Hydric balance, irrigation regime, center pivot.

* Autor para la correspondencia 


\section{INTRODUCCIÓN}

La papa ofrece altas producciones en un período vegetativo relativamente corto (70-90 días), siendo el principal cultivo de siembra de invierno en Cuba, ya que se adapta bien a nuestras condiciones climáticas. Para un buen desarrollo del cultivo de papas o patatas se requiere en promedio de 400 a 800 milímetros de agua dependiendo de las condiciones climáticas y de la duración del período vegetativo (Haverkort, 1986). Al respecto Bosnjak y Pejik (1996), reportan necesidades que varían entre 460 y 480 milímetros, en tanto que Klassen et al. (2001), señalan que para la obtención de buenos rendimientos y dependiendo de las condiciones climáticas de la zona, el cultivo de papas necesita de aproximadamente 455 milímetros de agua por temporada. El ciclo vegetativo del cultivo en condiciones de clima templado suele extenderse hasta 150 días, pero en Cuba normalmente no sobrepasa los 100 días, con frecuencia la mayoría de las variedades comerciales se cosechan a los 80-90 días (López et al., 1984). Este detalle marca la diferencia en cuanto a los volúmenes de agua necesarios en Cuba y en los países de clima templado. Ekanayake (1994), señala en términos generales que el cultivo de papas debe regarse a 0.35 bares de potencial tensiométrico del suelo, para mantener un micromedio bien irrigado. Al respecto Soboh et al. (2000), afirman que la tensión de humedad ideal para el cultivo es de 0.4 bares, o sea 40 centibares. En realidad la diferencia entre la opinión de ambos autores ( 5 centibares) resulta despreciable en la práctica del regadío. El riego es necesario en el país pues las precipitaciones durante el período seco del año no son suficientes para obtener un desarrollo adecuado de la mayor parte de los cultivos. Sin embargo, los suelos del "Valle del Yabú" no son los más adecuados para este cultivo por su alto contenido de arcilla, luego el cultivo de papas en los mismos reclama de especiales cuidados. El principal objetivo de este trabajo es generar una metodología para la programación del riego de la papa mediante información agrometeorológica.

\section{MATERIALES Y MÉTODOS}

En los dos años de estudio, la papa se plantó primero a inicios de enero/2009 y después a finales de diciembre/2009, fechas que se corresponden con el periodo seco., donde se presentan las más bajas temperaturas del aire. El suelo donde se realizaron las observaciones es pardo sin carbonato, plastogénico medianamente humificado con capacidad de campo de $50 \%$ s.s y densidad aparente de $1.0 \mathrm{~g} / \mathrm{cm} 3$. En la figura 1 se presentan las principales variables climáticas en la campaña 2009. La lluvia totalizo $58.3 \mathrm{~mm}$ en el ciclo del cultivo.

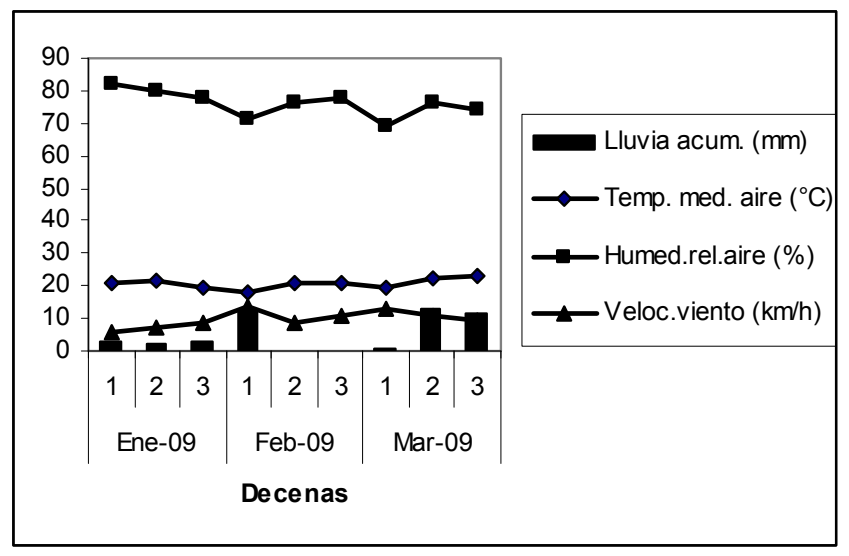

Figura 1. Principales variables climáticas en la cosecha 2009

En la figura 2 se presentan las principales variables climáticas durante la cosecha 2009-10. Allí puede apreciarse, que en las decenas finales del cultivo, esto es a finales de febrero y de marzo/10 se produjeron importantes lluvias anormales en estas fechas y que provocaron dificultades fitosanitarias al cultivo y después complicaron la cosecha. La lluvia totalizó 189.2 $\mathrm{mm}$ en el ciclo vegetativo del cultivo. En la tabla 1 se presentan las características de la máquina de riego utilizada en el campo 14 de la Unidad Cooperativa \# 2.

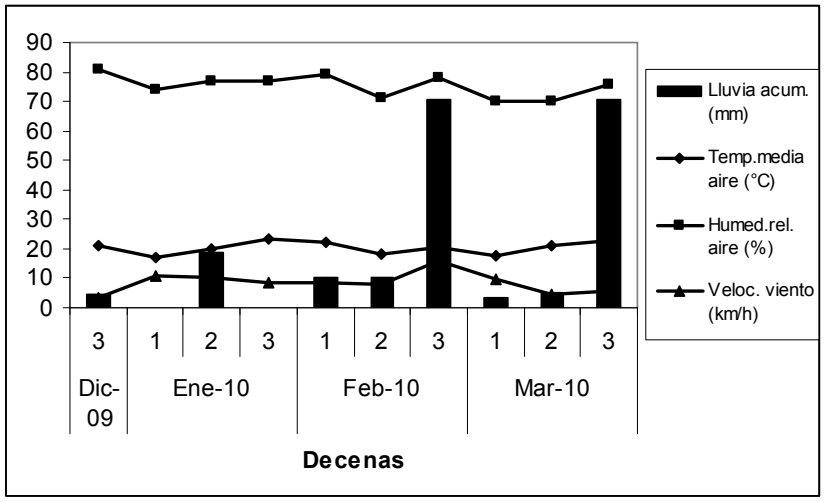

Figura 2. Principales variables climáticas en la cosecha 2009-10.

En la primera cosecha, se plantó la variedad Atlas con semilla fraccionada en toda el área y en la segunda 
cosecha se plantó la variedad Santana, con semilla fraccionada en la mitad del área.

Tabla 1. Características del pívot utilizado

\begin{tabular}{|c|c|c|c|}
\hline No. torres & Area regada (ha) & Caudal (l/s) & Longitud (m) \\
\hline 5 & 20.13 & 27.6 & 260 \\
\hline
\end{tabular}

En la primera cosecha, se plantó la variedad Atlas con semilla fraccionada en toda el área y en la segunda cosecha se plantó la variedad Santana, con semilla fraccionada en la mitad del área.

Se ubicaron tres tensiómetros a $20 \mathrm{~cm}$ y otros tres a $40 \mathrm{~cm}$ de profundidad, los primeros decidían el riego entre 40-50 centibares y los segundos para chequear la profundidad donde se almacenaba el agua de riego y de lluvia. La programación del riego, se monitoreó también según el balance hídrico agrometeorológico, a partir de calcular la evapotranspiración de referencia (Eto) por la formula de Penman-Monteith (Allen et al., 1998), el uso de los coeficientes Kc ajustados según el desarrollo del cultivo, las propiedades hidrofisicas del suelo y la medición correcta de las precipitaciones y las láminas de riego aplicadas. Los valores de tensión de humedad y de las muestras de humedad del suelo, se usaron para calibrar el balance agrometeorológico, el cual, una vez bien ajustado será el método que prevalezca para la programación del riego en la zona, método éste hoy validado en la práctica del regadío mundial Tarjuelo et al., (2002) y Carlesso et al., (2007).

\section{RESULTADOS Y DISCUSIÓN}

Los resultados de la evaluación pluviométrica de la máquina realizados en la primera campaña aparecen en la tabla 2, los realizados en la campaña siguiente no se diferencian de estos, por eso no se ofrecen.

Tabla 2. Resultados de la evaluación pluviométrica de la maquina

\begin{tabular}{|l|c|}
\hline \multicolumn{1}{|c|}{$\begin{array}{c}\text { Parámetros de la } \\
\text { evaluación. }\end{array}$} & $\begin{array}{c}\text { Fase de Brotación y } \\
\text { Establecimiento }\end{array}$ \\
\hline $\begin{array}{l}\text { Lámina de agua requerida } \\
(\mathrm{mm})\end{array}$ & 12.09 \\
\hline $\begin{array}{l}\text { Regulación de la máquina } \\
(\%)\end{array}$ & 16 \\
\hline $\begin{array}{l}\text { Velocidad de la última torre } \\
(\mathrm{m} / \mathrm{min} .)\end{array}$ & 1.2 \\
\hline $\begin{array}{l}\text { Lámina Media Ponderada } \\
\text { Aplicada (mm) }\end{array}$ & 12.3 \\
\hline
\end{tabular}

\begin{tabular}{|l|c|}
\hline $\begin{array}{l}\text { Coeficiente de Uniformidad } \\
\text { Ponderada }\end{array}$ & $86.7 \%$ \\
\hline
\end{tabular}

Los resultados demuestran que la maquina aplica el agua con buena uniformidad y entrega la lamina que señala el cronometro con bastante precisión. Sin embargo, cerca del pívot aplica volúmenes demasiado altos como se observa en la figura 3, la cual, muestra la pluviometría y la media ponderada en toda su longitud según el procesamiento que realiza el software "Pluviopivot" de Pacheco y Pacheco (2004).

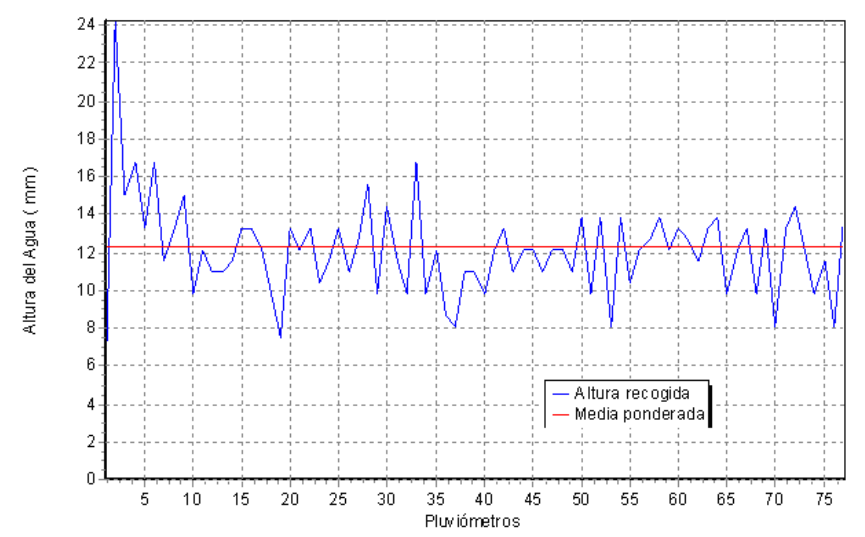

Figura 3. Evaluación pluviométrica de la máquina de riego.

En las dos cosechas se estudió la profundidad de las raíces por fases del cultivo y se determinó que el agua de riego debe almacenarse hasta $25 \mathrm{~cm}$ de profundidad, lo que queda explicito en la tabla 3. Más allá de $25 \mathrm{~cm}$, las características arcillosas del suelo no favorecen el crecimiento radicular. La programación de los riegos en la cosecha 2009 (tabla 4) se caracterizó por la aplicación de láminas de aguas muy pequeñas que no humedecían la zona radical del cultivo y esa alta frecuencia obstaculizaba los tratamientos fitosanitarios y culturales. Esa era la práctica corriente en todas las cooperativas del "Valle del Yabú".

Tabla 3. Capa activa y lámina de riego por fases.

\begin{tabular}{|l|c|c|}
\hline \multicolumn{1}{|c|}{ Fase de desarrollo } & $\begin{array}{c}\text { Capa } \\
\text { Activa (cm) }\end{array}$ & $\begin{array}{c}\text { Lamina de } \\
\text { riego neta } \\
\text { (mm) }\end{array}$ \\
\hline Plantación-Emergencia & 20 & 20 \\
\hline Crecimiento-Tuberización & 25 & 25 \\
\hline $\begin{array}{l}\text { Engrosamiento de los } \\
\text { tubérculos }\end{array}$ & 25 & 25 \\
\hline Maduración & 25 & 25 \\
\hline
\end{tabular}


En la campaña 2009-10 la dirección cooperativa comenzó a aplicar láminas de riego mayores según el suelo y fase fonológica del cultivo (tabla 5), pero a partir de fuertes precipitaciones que se presentaron a mediados de febrero y causaron pudriciones de tubérculos en dos cuadrantes por falta de drenajes, la dirección del riego redujo las laminas de riego con lo cual, el cultivo como se verá más adelante, padeció de estrés hídrico durante el mes de marzo.

Tabla 4. Resultados de la programación de riego en el 2009

\begin{tabular}{|c|c|c|c|c|}
\hline $\begin{array}{l}\text { No de } \\
\text { de los } \\
\text { riegos }\end{array}$ & $\begin{array}{c}\text { Fecha } \\
\text { de la } \\
\text { activida } \\
\text { d }\end{array}$ & $\begin{array}{c}\text { Interval } \\
\text { o entre } \\
\text { riegos } \\
\text { (días) }\end{array}$ & $\begin{array}{c}\text { Lámina } \\
\text { de agua } \\
\text { bruta } \\
\text { aplicada } \\
\text { por la } \\
\text { máquina } \\
\text {. (mm) }\end{array}$ & $\begin{array}{c}\text { Fase } \\
\text { fenológica } \\
\text { del cultivo. }\end{array}$ \\
\hline 1 & 4-1-09 & & 4.83 & \\
\hline 2 & $5-1-09$ & 1 & 4.83 & $\begin{array}{c}\text { Plantación } 6 \\
\text { Enero }\end{array}$ \\
\hline 3 & $10-1-09$ & 4 & 6.04 & \\
\hline 4 & 14-1-09 & 4 & 6.04 & \\
\hline 5 & 18-1-09 & 4 & 6.04 & \\
\hline 6 & 21-1-09 & 3 & 25 & \\
\hline 7 & $26-1-09$ & 5 & 25 & Emergencia \\
\hline 8 & 6-2-09 & 9 & 25 & \\
\hline 9 & 14-2-09 & 8 & 25 & $\begin{array}{l}\text { Crecimiento- } \\
\text { Tub. }\end{array}$ \\
\hline 10 & 16-2-09 & 2 & 25 & \\
\hline 11 & $21-2-09$ & 5 & 12.09 & \\
\hline 12 & $26-2-09$ & 6 & 12.09 & \\
\hline 13 & 2-3-09 & 3 & 6.04 & $\begin{array}{c}\text { Engrosamien } \\
\text { to }\end{array}$ \\
\hline 14 & $4-3-09$ & 2 & 25 & \\
\hline 15 & 7-3-09 & 2 & 6.04 & \\
\hline 16 & 9-3-09 & 2 & 16.11 & \\
\hline 17 & $12-3-09$ & 3 & 6.04 & \\
\hline 18 & 14-3-09 & 2 & 25 & \\
\hline 19 & 26-3-09 & 12 & 6.04 & Maduración \\
\hline 20 & $30-3-09$ & 3 & 8.06 & \\
\hline 21 & $2-4-09$ & 3 & 8.06 & \\
\hline 22 & $6-4-09$ & 4 & 8.06 & \\
\hline 23 & $9-4-09$ & 3 & 12.09 & \\
\hline $\begin{array}{l}\text { Media/ } \\
\mathrm{T}\end{array}$ & & 3.5 & $\begin{array}{l}225 \mathrm{~m} \\
\text { brutos(20 } \\
2 \mathrm{~mm} \\
\text { netos) } \\
\text { desde } \\
\text { brotación }\end{array}$ & \\
\hline
\end{tabular}

En las tablas 4 y 5 puede apreciarse, que en la cosecha 2009 se aplicaron $202 \mathrm{~mm}$ de agua de riego y en la del 2009-10 solamente $148 \mathrm{~mm}$ en ambos casos a partir de la brotación y como láminas netas.

Tabla 5. Resultados de la programación en el 2010.

\begin{tabular}{|c|c|c|c|c|}
\hline $\begin{array}{l}\text { No de } \\
\text { riegos }\end{array}$ & $\begin{array}{c}\text { Fecha } \\
\text { de } \\
\text { riego. }\end{array}$ & $\begin{array}{l}\text { Intervalo } \\
\text { entre } \\
\text { riegos } \\
\text { (días) }\end{array}$ & $\begin{array}{c}\text { Lámina } \\
\text { de agua } \\
\text { bruta } \\
\text { aplicada } \\
\text { por la } \\
\text { máquina. } \\
\text { (mm) }\end{array}$ & $\begin{array}{c}\text { Fase } \\
\text { fenológica del } \\
\text { cultivo. }\end{array}$ \\
\hline & $\begin{array}{l}(11- \\
26)- \\
\text { Dic- } \\
2009\end{array}$ & Mine* & 48.4 & \\
\hline 1 & $\begin{array}{c}06- \\
01- \\
2010\end{array}$ & - & 22.2 & $\begin{array}{l}\text { Plantación- } \\
\text { brotación }\end{array}$ \\
\hline 2 & $\begin{array}{c}13- \\
01- \\
2010 \\
\end{array}$ & 6 & 22.2 & \\
\hline 3 & $\begin{array}{c}25- \\
01- \\
2010\end{array}$ & 11 & 27.8 & $\begin{array}{c}\text { Crec- } \\
\text { tuberización }\end{array}$ \\
\hline 4 & $\begin{array}{c}11- \\
02- \\
2010\end{array}$ & 16 & 27.8 & \\
\hline 5 & $\begin{array}{c}17- \\
02- \\
2010\end{array}$ & 5 & 16.11 & Engrosamiento \\
\hline 6 & $\begin{array}{c}05- \\
03- \\
2010\end{array}$ & 15 & 16.11 & \\
\hline 7 & $\begin{array}{c}10- \\
03- \\
2010\end{array}$ & 4 & 12.09 & \\
\hline 8 & $\begin{array}{c}18- \\
03- \\
2010 \\
\end{array}$ & 7 & 9.67 & Maduración \\
\hline 9 & $\begin{array}{c}20- \\
03- \\
2010\end{array}$ & 1 & 9.67 & \\
\hline Media & & 7.2 & $\begin{array}{c}\text { (148 mm } \\
\text { (neto) }\end{array}$ & \\
\hline
\end{tabular}

En la cosecha 2009-10, la dirección de riego de todas las cooperativas del "Valle del Yabú" conscientes de la evaluación realizada en la cosecha 2009, orientaron incrementar las láminas de riego en todas las máquinas y 
alargar los intervalos entre riegos, con lo cual, no hubo maquinas atascadas y se realizaron con mayor calidad los tratamiento fitosanitarios y labores culturales. Esta medida bajó el promedio de riegos a la papa en 10 maquinas de pivote central de 24 a 13 al comparar la cosecha 2009 con la de 2010, con lo cual, también se ahorró energía eléctrica.

La variación de las tensiones de humedad en el suelo durante el mes de marzo/09, en el cual, los tubérculos estaban en la fase de engrosamiento puede verse en la figura 4 donde se aprecia que a partir del día 18, las tensiones a $20 \mathrm{~cm}$ de profundidad eran altas y con valores que en la profundidad de $40 \mathrm{~cm}$ (capa esta usualmente húmeda) estaban por encima de 50 centibares.

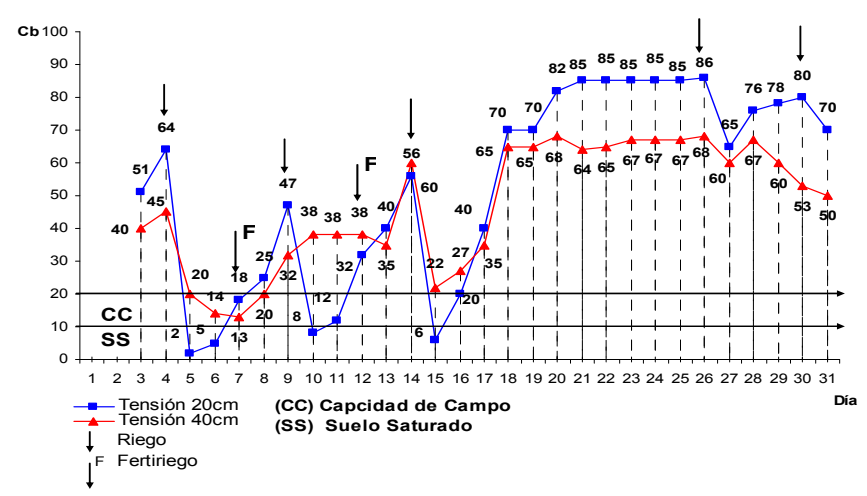

Figura 4. Variación de la tensión de humedad del suelo a 20 y $40 \mathrm{~cm}$ de profundidad en marzo/09

Luego, cuando se aplicaron riegos, los volúmenes de agua fueron muy pequeños y provocaron muy pobre reacción de la tensión a 20 y $40 \mathrm{~cm}$ de profundidad. También se aprecia que los fertirriegos aportaron muy poca agua al suelo, mientras que generaron vueltas adicionales de la máquina. Un prolongado déficit hídrico se observa por riegos pequeños también en marzo 2010 (figura 5), por las razones ya explicadas. Esta práctica de aplicar láminas de riego pequeñas durante el mes de marzo, sin reponer el déficit existente en el suelo, causó importante déficit hídrico en el período de engrosamiento de los tubérculos, que debió afectar los rendimientos.

En ambas cosechas de papa se realizó el balance hídrico agrometeorológico diario para la programación del riego y fue calibrado con muestras de suelo y el uso de tensiómetros. El balance hídrico agrometeorológico demostró estar en condiciones, de ser el futuro de la programación del riego en nuestro país.

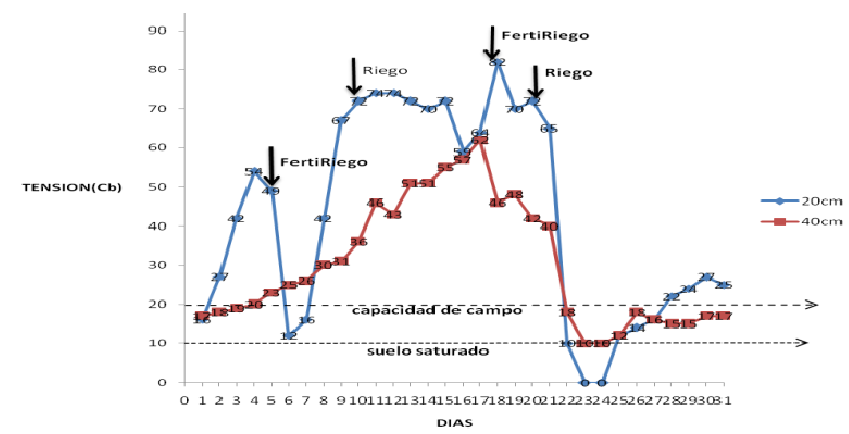

Figura 5.Variación de la tensión de humedad del suelo a 20 y $40 \mathrm{~cm}$ de profundidad en marzo/10

En el 2009 se obtuvo un rendimiento de 30.57 t/ha de tubérculos y en el 2009-10 las fuertes lluvias de febrero provocaron pudriciones por falta de drenaje en este suelo pesado reduciendo el rendimiento medio de la máquina de riego a $19.45 \mathrm{t} / \mathrm{ha}$, pero se realizó una cosecha en microparcelas en el cuadrante 3 del área, el cual, no fue atacado por enfermedades y se obtuvo un rendimiento de $35.75 \mathrm{t} / \mathrm{ha}$, lo que demuestra las potencialidades que existían sin las afectaciones ocasionadas por el mal drenaje.

El rendimiento real, también fue reducido en el 2009-10 por un fuerte ataque de pulgones y ácaros en el periodo de maduración que no permitió traslocar nutrientes de las partes aéreas a los tubérculos. Lo anterior fue demostrado mediante dos sucesivos análisis de muestras de masa seca de los tubérculos en el periodo de maduración con 15 días de diferencia, (15 de marzo y $1^{\text {ro }}$ de abril) donde no se encontró incremento de la masa seca, la cual, se mantuvo en $22.16 \%$.

\section{CONCLUSIONES}

Las láminas de agua aplicadas (10-12 $\mathrm{mm})$ a la papa en el suelo estudiado no humedecen suficientemente la zona radical del cultivo y acumulan grandes déficit de humedad. El número de riegos que necesita el cultivo en todo su ciclo en la zona en estudio, con láminas de agua de 20-25 mm, dependerá del comportamiento del año climático y época de plantación y oscilará entre 12-16. El método de balance hídrico agrometeorológico diario con los coeficientes Kc ajustados, demostró ser adecuado para la programación del riego de la papa. 


\section{REFERENCIAS}

Allen, R. G., Pereira, L., Raes, D y Smith, M. (1998). Crop Evapotranspiration. Guidelines for computing crop water requirements. FAO Irrigation and Drainage paper No. 56. Rome, Italy.

Bosnjak, D. Y Pejik, B. (1996). Potatoes water requirement in the Chernozem zone of Yugoslavia. Acta Horticulturae, Leuven, [en línea]: documento electrónico fuente en internet. V.1, n.449. pp 211-215 [Consulta: 01-03-10] Disponible en: http://www.actahort.org/books/449/449 29.htm.

Carlesso, R., Petry, M y Trois. C. (2007). Rede de estacoes meteorológicas automáticas para prover a necessidade de irrigacao das culturas. En: Taller Internacional: Modernización de Riegos y Uso de Tecnologías de Información. ISBN 13:978-92-9039881-3. La Paz, Bolivia.

Ekanayake, I. (1994). Estudios sobre estrés por sequía y necesidades de riego en la papa. Guía de Investigación p. 40, CIP 30. Centro Internacional de la Papa.. Lima, Perú.

Havercort, A.J. (1986). Manejo de agua en la producción de papa. Boletín de Información Técnica 15 CIP. p. 24. Editorial Agropecuaria Hemisferio Sur. Centro Internacional de la Papa. Lima, Perú.

Klassen, G., Mills, G. Y Geisel, B. (2001). Potato Production. Irrigation. [en línea]: documento electrónico fuente en internet [consulta: 05-01-2010]. Disponible en:

http://www.gov.mb.ca/agriculture/crops/potatoes/bda01s $\underline{11 . h t m l}$.

López, M., Vázquez, E. y López, R. (1984). Raíces y tubérculos. P. 304, Editorial Pueblo y Educación.

Pacheco, y Pacheco, J. (2004). Aplicación de software para calcular coeficientes de uniformidad ponderados por superficie en máquinas de pivote central. Revista EIDENAR, Año 1, Vol.2. Escuela de Ingeniería de Recursos Naturales y del Ambiente. Universidad del Valle, Cali, Colombia.
Soboh, G., Sully, R. y Hopkins, H. (2000). Mecanismos para incrementar el número de tubérculos. Boletín de la Papa, Rede Papa. Vol.3, nº, 28 de febrero, 2001. De: Australian Potato Research. (31 de julio-02 de agosto, 2000, Adelaide, Australia). Development and Technology Transfer Conference. [Consulta: 08-022010]. Disponible en: http://www.redepapa.org/boletintreintacuatro.html

Tarjuelo J. M., Juan J. A., Ortega J. F., Álvarez N. (2002). Irrigation Advisory Services and Participatory Extension in Irrigation Management for farmers (SIAR) in Castilla la Mancha: Action and Limitations, Workshop organized by FAO - ICID, Montreal, Canada

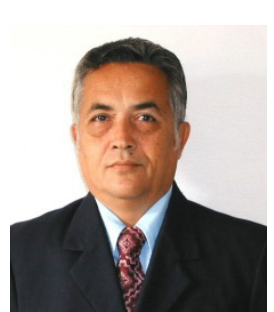

Juan Pacheco Seguí se graduó de Ingeniero Agrónomo en Abril de 1974 en la Universidad Central de Las Villas en Cuba. Su área de atención es el Riego y Drenaje.

Obtuvo el grado de Doctor en Ciencias Agrícolas en el Instituto de Hidrotecnia y Mejoramiento de Tierras de Sofía, Bulgaria en 1983. En 1986 asistió al 25th Curso Internacional de Drenaje Agrícola del ILRI en Wageningen, Holanda. Realizó entre los años 2006-07 una estancia Posdoctoral por cuatro meses en la Universidad Federal de Santa Maria, R. S-Brasil. Dirige proyectos encaminados a crear Sistemas de Asesoramiento a la Programación del Riego en Regiones Agrícolas. 